\title{
Multi-pose Face Detection under Strong Light Condition for Android Driver Fatigue detection System
}

\author{
Guangmin Sun, Qing Zhang, Junjie Zhang \\ Department of Electronic Engineering, Beijing University of Technology, Beijing, China \\ gmsun@bjut.edu.cn, sunnydayzq@aliyun.com
}

Keyword: Android fatigue detection, face detection, AdaBoost

\begin{abstract}
Fatigue driving, a hot issue in the field of transportation research, has become a worldwide problem which always leads to traffic accident. Existing detection method always based on costly hardware platform sensors. By developing an android APP using image processing algorithm, we can lower the costs and enable convenient detection. Generally, face detection is a key step in fatigue drive detection. However, correct result can't be required by Android system detection API, especially in side face detection or strong light condition. A smart-phone based algorithm is presented in this paper to deal with side face situation and detection under strong light. Specially, the algorithm is based on a number of AdaBoost classifier that can distinguish different poses, and it exploits the color skin model with illumination compensation to increase detection quality.
\end{abstract}

\section{Introduction}

Fatigue driving, along with drunk driving, has become a major factor in traffic accidents. The figures from The National Highway Traffic Safety Administration (NHTSA) showed that around $25 \%$ traffic accidents caused by driver's fatigue[1].Therefore, it is necessary to examine the drive's driving state and sending out warning message while fatigue. Several car manufacturers have already equipped their cars with drowsiness detection systems to ensure the driver's safety. However, these systems are not convenient and universal enough for public use. Nowadays, smart phone become more and more popular, a few smartphone-based fatigue detection systems have been developed for IOS or Android devices. B. Lee et. al using a data fusion based on eye features, bio-signal variation and some vehicle features to measure drivers' safety levels[2]. Generally, face detection is a key step to evaluate eye features and detect driver fatigue. However, Android system doesn't have a reliable face detection API. These API not only can't determine side face situation but also provide inaccurate detection results under strong light conditions. In this paper, we present a smartphone-based face detection algorithm. Using Adaboost classifier and skin color model, this algorithm can achieve real-time face detection with high accuracy. It is convenient for drivers to use, for only one smartphone with front camera needed, and easy to promote further access on fatigue detection system.

\section{Face Detection under Strong Light}

In order to get better result of drowsiness detection, real-time and steady face detection is required for Android system. Skin color model is applied for this project, it suits well for complex situations such as head rotation and different expressions with relative stability.

A. Color Space Selection

Basically, there are three kinds of commonly used color space: RGB, HIS, YCbCr[3]. Generally, different segmentation results will be required under different color space, we choose YCbCr color space due to the segment results under strong light. 


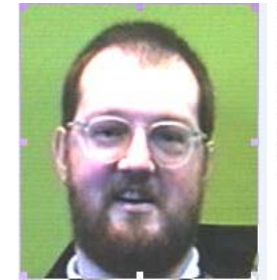

(a)Original image

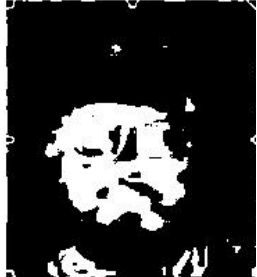

(b)RGB

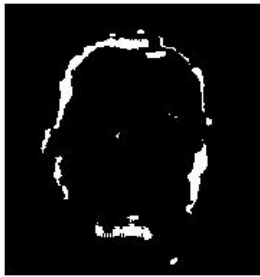

(c)HSI

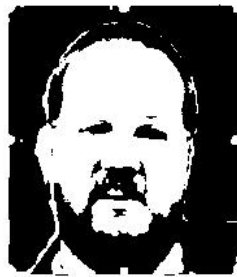

(d) $\mathrm{YCbCr}$

Fig. 2. Segment under different color space

\section{B. Conversion and threshold segmentation}

Generally, RGB color space is the most commonly used color space, color space conversion is a must before threshold segmentation. Specific conversion formula on our system is as follows:

$$
\left\{\begin{array}{l}
Y=0.273 R+0.512 G+0.108 B+18 \\
C b=-0.169 R-0.328 G+0.5 B+128 \\
C r=0.497 R-0.408 G-0.08 B+128
\end{array}\right.
$$

Then the parameters of YCbCr model which belong to skin color should be confirmed, according to $\mathrm{M}$. Hu, S. Worrall's research[4], values which meet the following requirements should be considered to belong to human skin.

$$
\left\{\begin{array}{l}
137<C r<177 \\
77<C b<127 \\
190<C b+0.6 C r<215
\end{array}\right.
$$

In the above formula, $\mathrm{Cb}, \mathrm{Cr} \in[0,255]$.

\section{Lighting Compensation under strong light}

A Special kind of Self-adaptive segmentation is used to improve the result of segment. Firstly, using the simple threshold get the sample of skin color image of human face, the threshold is shown as formula 2, then modeling double Gaussian model of skin color, luminance and chrominance of the image are separated to build GMM(Gaussian mixture model). GMM can effectively fit skin color area and suit for strong light condition. Basically, the probability density function of $\mathrm{CbCr}$ components is shown below:

$$
\rho(C b, C r)=\sum_{i=1}^{4} \pi \cdot \frac{1}{(2 \pi)^{\frac{d}{2}}\left|\Sigma_{i}\right|} \bullet \exp \left(-\frac{1}{2}\left(\mu-\mu_{i}\right) \Sigma_{i}\left(\mu-\mu_{i}\right)^{T}\right)
$$

Where $\mu=(C b, C r), \mu_{i}=\left(C b_{i}, C r_{i}\right), C b_{i}=\frac{1}{N} \sum_{j}^{N} C b, \Sigma_{i}=\frac{1}{N} \sum_{j}^{N}\left(\mu-\mu_{i}\right)^{T}\left(\mu-\mu_{i}\right), N$ means the total number of skin color pixels.

After building the GMM, similarity measure between pixels and SGM (single Gaussian model) is conducted with mahalanobis distance, then segment the binary image of $\mathrm{Y}, \mathrm{CbCr}$ and last segment results is mixed together for better image segmentation results. Formula of similarity measure is as follow:

$$
D(x, S G M)=\sum_{i=1}^{h} \mu_{i} \sqrt{\left(x-m_{i}\right)^{T} \Sigma_{i}^{-1}\left(x-m_{i}\right)}
$$

Where $h, \mu_{i}, m_{i}, \Sigma_{i}$ means number of SGM, weight, average, covariance of SGM.

Results are shown in figure 3 :

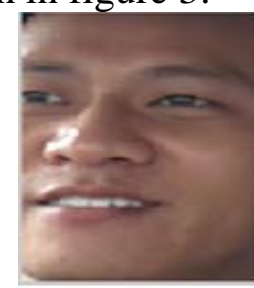

(a)Original image

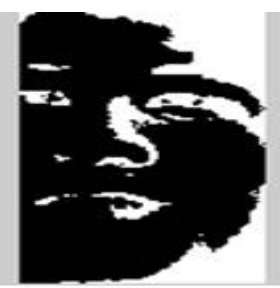

(b)threshold segmentation

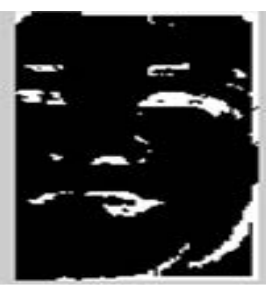

(c)after compensation

Fig. 3. Different skin color segmentation result 


\section{Multi-pose Face Detection}

Considering the multi-pose problem of drivers, an sample training algorithm called Adaboost algorithm is needed [5],the basic idea of Adaboost algorithm is eigenvalue calculation, training multiple weak classifier and then form strong classifier. Adaboost algorithm and skin color model are combined together in this project for real-time use. Firstly scope of the test is narrowed with color information, then multiple Adaboost face detector is applied to improve the detection speed and reduce the probability of error check.

A. sample training

There is no common color facial library can be used, our face database is obtained from personal video, including front face, side face, face look up and down. Database is as follow:

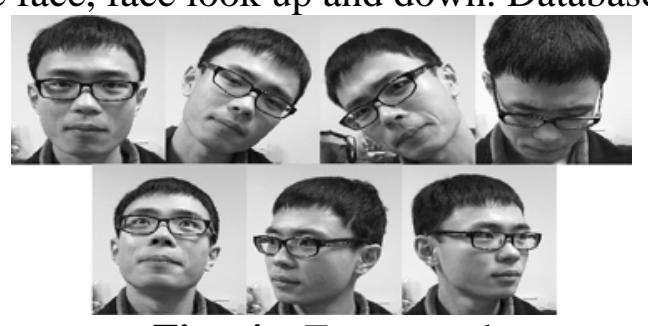

Fig. 4. Face sample

These face samples are collected from android smartphone in the indoor light environment, including 2684 sampled of different skin color and posture, screen resolution is $640 \times 480$, and the resolution of face sample is $160 \times 180$.

Non-face samples including different images like animals, plants and mountain views and so on, a total of 4130 samples, both are $640 \times 480$ in size.

All the samples after simple preprocessing like histogram equalization and grayscale conversion, the highest error detection probability $F_{\text {max }}$ is confirmed as 0.05 , and error detection probability of strong classifier $f_{\max }$ should be confirmed either. The whole system consists of at least $\log _{f_{\max }} F_{\max }$ strong classifiers.

\section{B. Face Detection}

Generally, Adaboost algorithm use rectangle features to extract features, rectangle features is defined as the difference of sum of all pixels between two different rectangular area, here are four basic Haar-like feature.

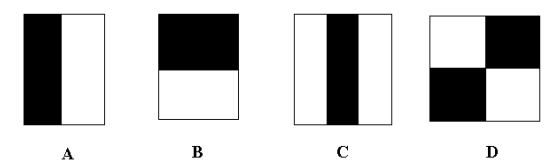

Fig. 5. Rectangular feature temples

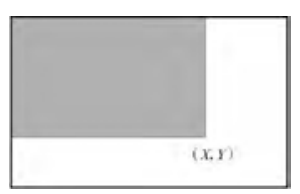

Fig. 6. Vertical rectangular integral image Integral image of rectangular feature in an image I is defined as figure 6.

$$
c(x, y)=\sum_{x_{1} \leq x} \sum_{y_{1}} I\left(x_{1}, y_{1}\right)
$$

$\mathrm{C}(\mathrm{x}, \mathrm{y})$ represents number of rectangular features at the top-left corner.

In order to make use of the skin color feature, skin color binary operations is needed, the basic flow chart is shown below.

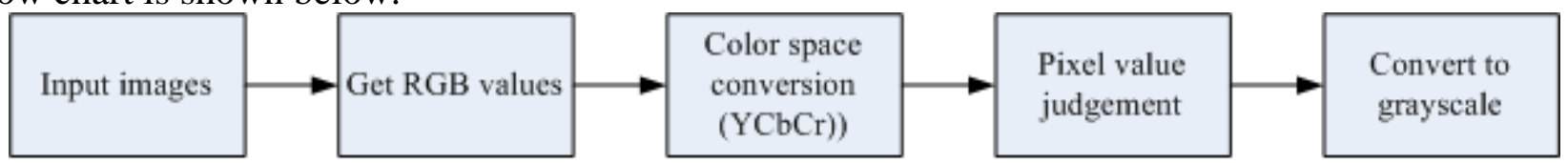

Fig. 7. Binary conversion

Formula 1 is used for color space conversion. After conversion, when formula 2 is satisfied, the pixel is considered to be skin color pixel, then the value of R,G,B in image is turned into 255, the value of R,G,B is 0 when don't meet the requirements.

When the binary conversion is finished, all the pixels in the picture should be judged if the pixel number is 255, and then the pixel number added to the total number. Here we introduce the concept of integral figure to improve the speed of our algorithm, the formula is described in the above, color 
number take the place of rectangle feature, then the total number of skin color pixels is obtained in an $\mathrm{w} \times \mathrm{h}$ image:

$$
\text { cnum }=c(x, y)+c(x-w, y-h)-c(x-w, y)-c(x, y-h)
$$

In a fatigue detection image, most of the pixels are skin color pixels, so percentage of skin color pixel could be used as an indicator of human face image. The average percentage is unlikely to be $100 \%$ due to the existence of the hair, according to our experiments; the percentage is $44 \%$ to $92 \%$. So non-face image can quickly be ruled out.

\section{Algorithm transplant and results}

Because Android system is built on Java language, further work need to be done with the algorithm transplant.

In Android system, our algorithm written by $\mathrm{C} / \mathrm{C}++$ language is called the native code, system environment of Android NDK, SDK and Eclipse IDE should be configured first. After the configuration, Android.mk file will be compiled, our NDK version is android-ndk-r9.

After compiling the file, project dictionary automatically create a folder /libs/armeabi to put the .so file, this .so file can be loaded to finish the transplant.

Detection results are shown by the following image:

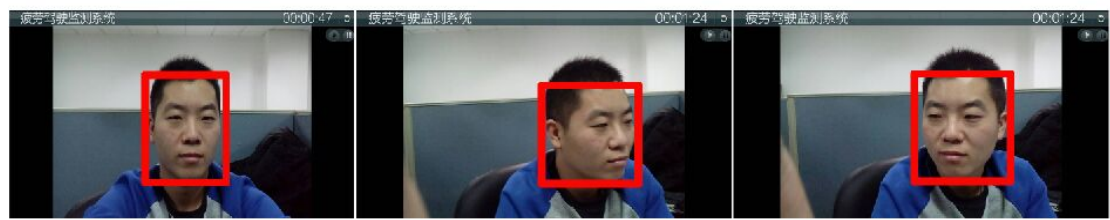

Fig. 8. GUI and results

\section{Conclusion}

A version-based face detection for Android system is presented in this paper, and this algorithm solve the multi-pose and strong light problem, making full use of Adaboost algorithm and skin color model. According to the results of our experiment, this algorithm can effectively used in Android system, and don't need any hardware sensors, also the detection results is much better than the Android system API.

\section{Acknowledgments}

This work was supported by National Natural Science Foundation of China (20222201 and 61305026) and Beijing Municipal Commission of Education (KM200710005009, PXM2009_014204_09_000154 and KM201310005006).

\section{References}

[1] J. D. Lee, K. L. Young, and M. A. Regon, Defining driver distraction, in Driver Distraction: Theory, Effects and Mitigation. Boca Raton, FL: CRC, 2009.

[2] B. Lee, W. Chung, A smartphone-based driver safety monitoring system using data fusion, in Sensors, vol. 12, issue 12, 2012, pp. 17536-17552

[3] Singh, D S Chauhan, M. Vatsa and R. Singh. A robust skin color based face detection algorithm. Journal of Science \& Engineering 6(2003):227-234.

[4] $\mathrm{Hu}, \mathrm{M}$., et al. Face feature detection and model design for 2D scalable model-based video coding. Visual Information Engineering, 2003. VIE 2003. International Conference on IET, 2003:125-128.

[5] Viola P., Jones M. J. Robust Real-Time Face Detection International [J]. Journal of Computer Vision, 2004, 57 (2) : 137-154. 